\title{
THE THIRD SMALLEST EIGENVALUE OF THE LAPLACIAN MATRIX*
}

\author{
SUKANTA PATI ${ }^{\dagger}$
}

\begin{abstract}
Let $G$ be a connected simple graph. The relationship between the third smallest eigenvalue of the Laplacian matrix and the graph structure is explored. For a tree the complete description of the eigenvector corresponding to this eigenvalue is given and some results about the multiplicity of this eigenvalue are given.
\end{abstract}

Key words. Laplacian matrix, algebraic connectivity, third smallest eigenvalue, tree.

AMS subject classifications. 05C50, 15A48

1. Laplacian matrices. Let $G$ be a connected simple graph with vertex set $V=\{1,2, \cdots, n\}$, edge set $E$ and let each edge be associated with a positive number, called the weight of the edge. The above graph is called a weighted graph. An unweighted graph is just a weighted graph with each of the edges bearing weight 1 . All the graphs considered are weighted and simple, unless specified otherwise; all the matrices considered are real. The weight $w(i)$ of a vertex $i$ is the sum of the weights of the edges incident with it. The Laplacian matrix $L$ related to this graph is defined as $L=\left(l_{i j}\right)$, where

$$
l_{i j}=\left\{\begin{array}{cl}
w(i), & \text { if } i=j, \\
-\theta, & \text { if }[i, j] \in E \text { and the weight of the edge is } \theta \\
0, & \text { otherwise }
\end{array}\right.
$$

There are many interesting results known about Laplacian matrices. We refer the reader to [14]-[16] for surveys on this topic. Many authors have studied the relationship between the eigenvector corresponding to the second smallest eigenvalue and the graph structure; see, e.g., [1]-[13]. Indeed our research is motivated by all these works. In this paper we explore the relationship between the eigenvector corresponding to the third smallest eigenvalue and the graph structure. There is another motivation for this work. The second smallest eigenvalue (known as algebraic connectivity) of the Laplacian matrix of an unicyclic graph is very much related to the 3 rd smallest eigenvalue of a spanning tree of the unicyclic graph [3, 17]. Thus while studying the algebraic connectivity of a unicyclic graph some information about the third smallest eigenvalue of a Laplacian matrix of a spanning tree might prove to be helpful.

Henceforth the multiplicity of the algebraic connectivity is assumed to be one (i.e., simple), unless stated otherwise. We will denote the third smallest eigenvalue of the

\footnotetext{
${ }^{*}$ Received by the editors on 23 February 2001. Final manuscript accepted for publication on 14 July 2001. Handling Editor: Stephen J. Kirkland.

${ }^{\dagger}$ Department of Mathematics, IIT Guwahati, North Guwahati, India, 781039 (pati@iitg.ernet.in). The work was done when the author was visiting the University of Regina, Saskatchewan, Canada. Research supported by the University of Regina and NSERC under grant numbers OGP0138251 and OGP22730700
} 
Laplacian matrix of a graph by ${ }^{3} \lambda$ and the corresponding eigenvector is referred to as a 3-vector of $L$. The term Fiedler vector will mean an eigenvector of $L$ corresponding to the algebraic connectivity.

2. Preliminary results. We notice that the smallest eigenvalue of $L$ is zero and the corresponding eigenvector is the all ones vector. Thus any other eigenvector is orthogonal to the all ones vector and contains at least one positive entry and at least one negative entry. It is known that the smallest eigenvalue 0 of $L$ is simple if and only if the graph $G$ is connected.

If $Y$ is a 3 -vector of $L$, then by the eigencondition at a vertex $v$ we mean the equation

$$
\sum_{(i, v) \in E} L(v, i) Y(i)=\left[{ }^{3} \lambda-L(v, v)\right] Y(v) .
$$

With respect to a vector $Z$, the vertex $v$ of $G$ is called a characteristic vertex of $G$ if $Z(v)=0$ and if there is a vertex $w$, adjacent to $v$, such that $Z(w) \neq 0$.

Note 2.1. If $v$ is a characteristic vertex of $G$ with respect to a 3-vector $Y$, then the eigencondition at $v$ implies that there are at least two vertices $u, w$ in $G$, adjacent to $v$ such that $Y(u)>0$ and $Y(w)<0$.

With respect to a vector $Z$, an edge $e$ with end vertices $u, w$ is called a characteristic edge of $G$ if $Z(u) Z(w)<0$. By $\mathcal{C}(G, Z)$ we denote the characteristic set of $G$ with respect to a vector $Z$, which is defined as the collection of all characteristic vertices and characteristic edges of $G$ with respect to $Z$.

Lemma 2.2. Let $G$ be connected and $Y$ be an eigenvector of $L$ corresponding to an eigenvalue $\lambda(\neq 0)$. Let $G_{1}$ be a connected subgraph of $G$ such that for each vertex $v \in G_{1}, Y(v)>0$ and for each vertex $w \notin G_{1}$ adjacent to any vertex in $G_{1}$, $Y(w) \leq 0$. Let $L_{1}$ denote the principal submatrix of $L$ corresponding to $G_{1}$. Then $\tau\left(L_{1}\right) \leq \lambda$, where $\tau\left(L_{1}\right)$ denotes the smallest eigenvalue of $L_{1}$. Further the inequality is strict if for some such vertex $w, Y(w)<0$.

Proof. With permutation similarity operations, we have

$$
L=\left[\begin{array}{c|c}
L_{1} & L_{12} \\
\hline L_{21} & L_{22}
\end{array}\right]\left[\begin{array}{l}
Y_{1} \\
\hline Y_{2}
\end{array}\right]=\lambda\left[\begin{array}{l}
Y_{1} \\
\hline Y_{2}
\end{array}\right],
$$

where $Y_{1}$ is the part of the eigenvector $Y$ corresponding to $G_{1}$. Thus from $L_{1} Y_{1}+$ $L_{12} Y_{2}=\lambda Y_{1}$, we have

$$
L_{1} Y_{1} \leq \lambda Y_{1},
$$

since $L_{12} Y_{2}$ is nonnegative. Here the inequality would be strict if $L_{12} Y_{2}$ is nonzero.

Recall that any eigenvector of $L$ which does not correspond to the eigenvalue 0 contains at least one positive entry and at least one negative entry. Thus $L_{1}$ is a proper principal submatrix of $L$ and hence is a nonsingular M-matrix. Thus the smallest eigenvalue of $L_{1}$ is positive and the corresponding eigenvector (say Z) is also positive (entrywise). Multiplying $Z^{T}$ from the left in equation (2.1) yields $\tau\left(L_{1}\right) \leq \lambda$. 
If there is a vertex $w \notin G_{1}$ adjacent to a vertex in $G_{1}$ such that $Y(w)<0$, then $L_{12} Y_{2}$ is nonzero and the strict inequality follows.

By a nonzero (zero, negative, positive) vertex $v$ of $G$ we mean a vertex of $G$ such that $Y(v) \neq 0(Y(v)=0, Y(v)<0, Y(v)>0$, respectively), where $Y$ is an eigenvector (which will be clear from the context) of $L$. A subgraph $H$ of $G$ containing a nonzero vertex of $G$ is called a nonzero subgraph of $G$. A subgraph $H$ of $G$ is called positive if each vertex of $H$ is positive. Negative subgraph is defined similarly. It is well known that if $G$ is a connected graph and $Y$ is a Fiedler vector (here the algebraic connectivity need not be simple) such that a cut vertex $v$ is a characteristic vertex and $G-\{v\}$ has at least two nonzero components, then for any other Fiedler vector $Z, v$ is also a characteristic vertex. In general the following can be said.

Lemma 2.3. Let $G$ be a connected graph and $Y$ be an eigenvector corresponding to an eigenvalue $\lambda(\neq 0)$ of the Laplacian matrix (here the algebraic connectivity need not be simple). Suppose that $v \in \mathcal{C}(G, Y)$ and $G-v$ has a positive component $B$. Then for any other eigenvector $Z$ corresponding to $\lambda, Z(v)=0$.

Proof. Note that since $v$ is a characteristic vertex and $G-\{v\}$ has a positive component, $v$ is thus a cut vertex. (This is simply because if $v$ is not a cut vertex then $G-\{v\}$ will have only one component and this component has to contain the negative and positive vertices adjacent to $v$.)

Also by the eigenvalue eigenvector relation and the fact that $Y(v)=0$, it follows that $\lambda$ is an eigenvalue of $L_{B}$ (this is the principal submatrix of $L$ corresponding to $B)$ corresponding to a positive eigenvector and hence $\tau\left(L_{B}\right)=\lambda$.

If $Z(v) \neq 0$ then let $Z(v)<0$. Consider the vector $X \equiv \epsilon Z+Y$, where $\epsilon$ is so small that $X(B)>0$. It is clear that we can choose such a vector $X$. Now applying Lemma 2.2 it follows that $\tau\left(L_{B}\right)<\lambda$, a contradiction.

We remark here that, in the above lemma, $v$ is not necessarily a characteristic vertex of $G$ with respect to $Z$. The following is well known [6].

Lemma 2.4. Let $G$ be a connected graph and $Y$ be a 3-vector of $L$. Then the subgraph induced by the vertices $v$ in $G$ for which $Y(v) \geq 0$ has at most 2 components (similarly the subgraph induced by the vertices $v$ in $G$ for which $Y(v) \leq 0$ has at most 2 components).

The following is proved in [1]. Here $\lambda_{-}(A)\left(\lambda_{+}(A)\right)$ denote the number of negative (positive) eigenvalues of $A$.

Proposition 2.5. Let $A=\left[\begin{array}{cc}B & C \\ C^{T} & E\end{array}\right]$ be a symmetric matrix, where $B, E$ are square. Let $U$ be a vector such that $B U=0$ and $C^{T} U \neq 0$. Then $\lambda_{-}(A) \geq \lambda_{-}(B)+1$.

Lemma 2.6. Let $G$ be a connected graph. Let $W$ be a set of vertices of $G$ such that $G-W$ is disconnected with at least 3 components. Let $G_{1}, G_{2}, G_{3}$ be three of the components of $G-W$ and $L_{1}, L_{2}, L_{3}$ be the corresponding principal submatrices of $L$. Suppose that $\tau\left(L_{1}\right) \leq \tau\left(L_{2}\right) \leq \tau\left(L_{3}\right)$. Then either $\tau\left(L_{3}\right)>{ }^{3} \lambda$ or $\tau\left(L_{2}\right)=\tau\left(L_{3}\right)={ }^{3} \lambda$, Thus, it is always true that $\tau\left(L_{3}\right) \geq{ }^{3} \lambda$.

We remember that the algebraic connectivity is always assumed to be simple throughout the paper.

Proof. It is sufficient to show that $\tau\left(L_{3}\right) \leq{ }^{3} \lambda \Rightarrow \tau\left(L_{3}\right)=\tau\left(L_{2}\right)={ }^{3} \lambda$. So, let $\tau\left(L_{3}\right) \leq{ }^{3} \lambda$ and first suppose that $\tau\left(L_{2}\right)<\tau\left(L_{3}\right)$. Let $W=\{1,2, \cdots, k\}$ and let 
$d_{i}=L(i, i), i=1,2, \cdots, k$. Let $L_{G-W}$ be the principal submatrix of $L$ corresponding to the graph $G-W$. After a permutation similarity operation we have

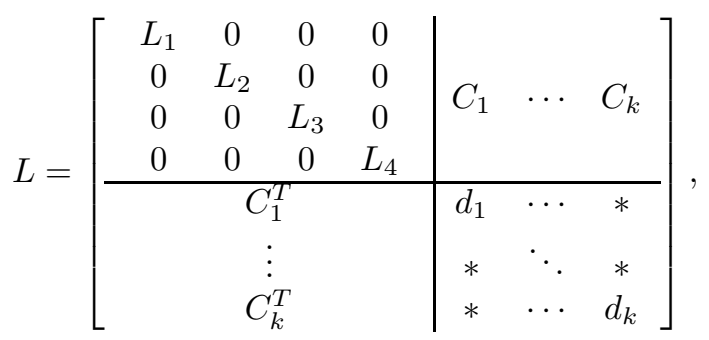

where the upper left block diagonal matrix in the above representation is $L_{G-W}$ and $L_{4}$ corresponds to the rest of the components of $G-W$ (that is those not equal to $\left.G_{1}, G_{2}, G_{3}\right)$. Let $U$ be the positive vector associated with $\tau\left(L_{3}\right)$. Consider the vector $U^{\prime}=\left[\begin{array}{llll}0 & 0 & U^{T} & 0\end{array}\right]^{T}$, where the zeros are added so that $L_{G-W} U^{\prime}=\tau\left(L_{3}\right) U^{\prime}$. Since at least one vertex in $W$ is adjacent to one of the vertices in $G_{3}$, there exists $i, 1 \leq i \leq k$, such that $C_{i}^{T} U^{\prime} \neq 0$. Note that $\lambda_{-}\left[L_{G-W}-\tau\left(L_{3}\right) I\right] \geq 2$, because of the hypothesis. Now, applying Proposition 2.5, we see that $\lambda_{-}\left[L-\tau\left(L_{3}\right) I\right] \geq 3$, and hence $\tau\left(L_{3}\right)>{ }^{3} \lambda$. This is a contradiction to the hypothesis that $\tau\left(L_{3}\right) \leq{ }^{3} \lambda$.

Next, let $\tau\left(L_{3}\right)=\tau\left(L_{2}\right)$. Since $L_{G-W}$ is a principal submatrix of $L$, using Cauchy interlacing theorem we get that the third smallest eigenvalue of $L$ is less than or equal to $\tau\left(L_{3}\right)$. But $\tau\left(L_{3}\right) \leq{ }^{3} \lambda$, by the hypothesis. So, we get $\tau\left(L_{3}\right)=\tau\left(L_{2}\right)={ }^{3} \lambda$.

As one of the applications of Lemma 2.6, we prove the following result.

Lemma 2.7. Let $G$ be a connected graph and $Y$ be a 3-vector of L. Let $W$ be a nonempty set of vertices of $G$ such that $Y(u)=0$, for all $u \in W$ and suppose that $G-W$ is disconnected with $t(\geq 3)$ nonzero components, $G_{1}, G_{2}, \cdots, G_{t}$. Let $L_{i}$ and $Y_{i}$ be the principal submatrix of $L$ and the subvector of $Y$ corresponding to $G_{i}$, respectively. Then the following occurs.

a. The multiplicity of ${ }^{3} \lambda$ is at least $t-2$. ( The multiplicity can be strictly larger than $t-2$, see Example 2.8.)

$b$. For at least $t-1$ indices $i, i \in\{1,2, \cdots, t\}, \tau\left(L_{i}\right)={ }^{3} \lambda$; for these indices $i$ the entries of each $Y_{i}$ are nonzero and of the same sign.

Thus the number of components containing both positive and negative vertices is at most 1 and if $F$ is such a component then the smallest eigenvalue of the corresponding principal submatrix of $L$ is less than ${ }^{3} \lambda$.

Proof. Let

$$
\hat{L}=\left[\begin{array}{cccc}
L_{1} & 0 & \cdots & 0 \\
0 & L_{2} & 0 & \vdots \\
\vdots & \vdots & \ddots & \vdots \\
0 & \cdots & 0 & L_{t}
\end{array}\right]
$$

For each $i=1, \cdots, t, L_{i} Y_{i}={ }^{3} \lambda Y_{i}$. So we have $\tau\left(L_{i}\right) \leq{ }^{3} \lambda$. So the $t$-th smallest eigenvalue of $\hat{L}$ is less than or equal to ${ }^{3} \lambda$. With a permutation similarity operation, 
we can assume that $\hat{L}$ is a principal submatrix of $L$. Thus applying Cauchy interlacing theorem we get that the $t$-th smallest eigenvalue of $L$ is also less than or equal to ${ }^{3} \lambda$. But since $t \geq 3$, we get that the $t$-th smallest eigenvalue of $L$ is equal to ${ }^{3} \lambda$. Thus the multiplicity of ${ }^{3} \lambda$ is at least $t-2$.

To prove b, suppose that $\tau\left(L_{1}\right) \leq \tau\left(L_{2}\right) \cdots \leq \tau\left(L_{t}\right)$. Then using Lemma 2.6, we get that $\tau\left(L_{i}\right)={ }^{3} \lambda, i=2,3, \cdots, t$. By Perron-Frobenius theory, the vectors $Y_{i}, i=2,3, \cdots, t$ are positive and unique up to a scalar multiple. $\square$

EXAMPLE 2.8 .

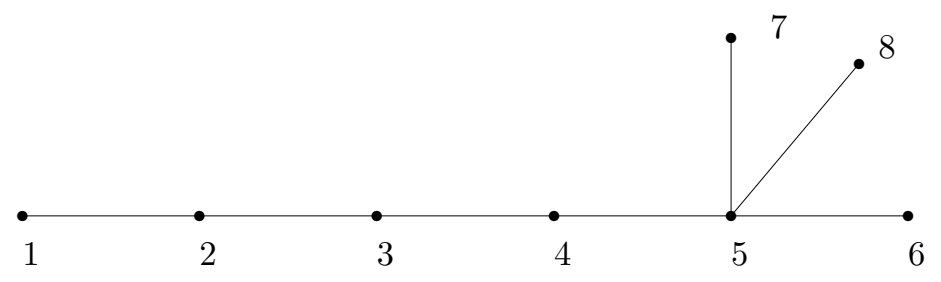

We consider an unweighted tree. The third smallest eigenvalue is 1 and a corresponding eigenvector is $Y=\left[\begin{array}{llllllll}0 & 0 & 0 & 0 & 0 & -1 & -1 & 2\end{array}\right]^{T}$. One can see that $G-W$ has one nonzero component if $5 \notin W$, otherwise $G-W$ has three nonzero components. The multiplicity of ${ }^{3} \lambda$ is 3 .

We remark here that from the proof of the above lemma, it is clear that under the assumptions of the lemma, if we have a nonzero component $H$ then $\tau\left(L_{H}\right) \leq{ }^{3} \lambda$.

The following is known for the algebraic connectivity (see for example [2]). Let $Y$ be a Fiedler vector for a tree and $v$ be any vertex. Let $T_{1}$ be a component of $T-v$ such that $\tau\left(L_{T_{1}}\right)<\mu$, where $\mu$ is the algebraic connectivity. Then $Y\left(T_{1}\right)$ is necessarily nonzero.

3. 3-vectors of a tree. In this section we give a complete description of a $3-$ vector of the Laplacian for an unweighted tree. To prove the main result we need some more preliminaries on 3 -vectors.

An $n \times n$ matrix $A$ will be called acyclic if it is symmetric and if for any mutually distinct indices $k_{1}, k_{2}, \cdots, k_{s}(s \geq 3)$ in $\{1,2, \cdots, n\}$ the equality

$$
A\left(k_{1}, k_{2}\right) A\left(k_{2}, k_{3}\right) \cdots A\left(k_{s}, k_{1}\right)=0
$$

is fulfilled. Thus the Laplacian matrix of a tree is acyclic.

Suppose that $Y$ is a 3 -vector of $L(T)$, where $T$ is an unweighted tree. Let us pose the following question. How many characteristic elements can $\mathcal{C}(T, Y)$ have ? The following proposition which is due to Fiedler (a part of theorem $(2,3)$ of [5]), supplies an answer to it.

Proposition 3.1. Let $A$ be $a n \times n$ acyclic matrix. Let $Y$ be an eigenvector of $A$ corresponding to an eigenvalue $\lambda$. Denote by $\omega_{+}$and $\omega_{-}$, respectively, the number of eigenvalues of $A$ greater than and less than $\lambda$. Let there be no "isolated" zero coordinate of $Y$, that is coordinate $Y(k)=0$ such that $A(k, j) Y(j)=0$ for all $j$. Then

$$
\omega_{+}=a^{+}+r, \quad \omega_{-}=a^{-}+r
$$




\section{ELA}

Sukanta Pati

where $r$ is the number of zero coordinates of $Y, a^{+}$is the number of those unordered pairs $(i, k)$ for which

$$
A(i, k) Y(i) Y(k)<0
$$

and $a^{-}$is the number of those unordered pairs $(i, k), i \neq k$, for which

$$
A(i, k) Y(i) Y(k)>0 \text {. }
$$

The following is an immediate corollary.

Corollary 3.2. Suppose that $T$ is an unweighted tree and $L$ its Laplacian matrix. Let $Y$ be a 3-vector. Then the number of characteristic elements in $\mathcal{C}(T, Y)$ is at most 2.

Proof. We know $L$ is an acyclic matrix. Observe that an "isolated" zero coordinate of $Y$ means a zero vertex of $T$ which is not a characteristic vertex. Here $\omega_{-}=2$. Let $\bar{T}$ be the graph obtained from $T$ by deleting those zero vertices of $T$ which are not characteristic vertices. Let $\bar{L}$ and $\bar{Y}$ be the principal submatrix of $L$ and the subvector of $Y$, respectively, corresponding to $\bar{T}$. It is clear that

$$
\mathcal{C}(T, Y)=\mathcal{C}(\bar{T}, \bar{Y}) .
$$

Also note that $\bar{L}$ is an acyclic matrix and ${ }^{3} \lambda$ is an eigenvalue corresponding to the eigenvector $\bar{Y}$. Since $\bar{L}$ is a principal submatrix of $L$,

$$
\bar{\omega}_{-} \leq \omega_{-}=2,
$$

where $\bar{\omega}_{-}$is the number of eigenvalues of $\bar{L}$ less than ${ }^{3} \lambda$. By Proposition 3.1,

$$
\bar{\omega}_{-}=a^{-}+r,
$$

where $r$ is the number of zero coordinates of $\bar{Y}$; that is, $r$ is the number of characteristic vertices in $\mathcal{C}(\bar{T}, \bar{Y})$. Also $a^{-}$is the number of those unordered pairs $(i, k), i \neq k$, for which $\bar{L}(i, k) \bar{Y}(i) \bar{Y}(k)>0$; that is, $a^{-}$is the number of characteristic edges in $\mathcal{C}(\bar{T}, \bar{Y})$. The result now follows in view of the above three equations.

In Example 2.8 we have already seen a tree $T$ and a 3-vector $Y$ of the Laplacian matrix such that the number of characteristic elements in $\mathcal{C}(T, Y)$ equals 1 , which is strictly less than 2 . In that example the multiplicity of the 3rd smallest eigenvalue was 2. It was proved by Fiedler that "if $T$ is an unweighted tree, $L$ the Laplacian matrix and $Y$ a 3-vector such that each entry of $Y$ is different from zero, then the number of characteristic elements in $\mathcal{C}(T, Y)$ is 2 and ${ }^{3} \lambda$ is simple"; see, [5, corollary $(2,5)]$. Thus one might wonder whether the cardinality of $\mathcal{C}(T, Y)$ can be 1 , given $Y$ is a 3-vector corresponding to a simple eigenvalue.

ExAmple 3.3. The tree in Figure 3.1 is unweighted. The eigenvalue ${ }^{3} \lambda=1$ is simple but $|\mathcal{C}(G, Y)|=1$, for the 3 -vector $Y=[0,0,0,0,1,-1]$.

Lemma 3.4. Let $T$ be a un weighted tree (here the algebraic connectivity need not be simple). Let $Y$ be an eigenvector of $L$ corresponding to the eigenvalue $\lambda(\neq 0)$. Let $[i, j]$ be an edge of $T$. Suppose that the component $T_{j}$ of $T-\{i\}$ containing $j$ is 


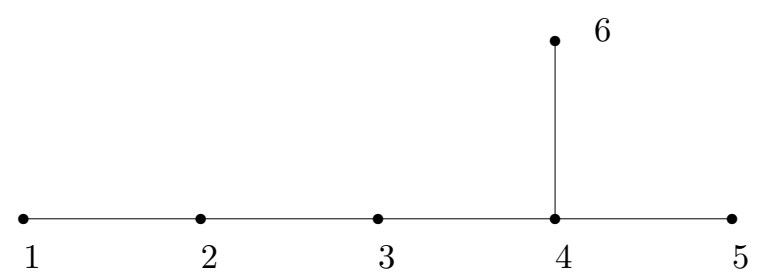

FIG. 3.1. ${ }^{3} \lambda$ is simple and $|\mathcal{C}(G, Y)|=1$.

positive. Then along any path that starts at $j$ and does not contain $i$ the entries of $Y$ increase and are concave down.

Proof. Consider the vector $U$ defined as: $U(s)=0$ if the vertex $s$ is not in $T_{j}$, otherwise $U(s)=1$. Now let us compute $U^{T} L Y$. On one hand it is $\lambda U^{T} Y=$ $\lambda \sum_{s \in T_{j}} Y(s)$. On the other hand it is $Z^{T} Y$, where $Z(i)=-1, Z(j)=1$ and $Z(s)=0$, if $s \notin\{i, j\}$. Thus $Z^{T} Y=Y(j)-Y(i)$. So we get the following equation.

$$
Y(j)-Y(i)=\lambda \sum_{s \in T_{j}} Y(s)
$$

Since the vector $Y$ is positive on $T_{j}$ it follows that $Y(j)>Y(i)$. Further if $[j, k]$ is an edge in $T_{j}$ then a similar argument will show that

$$
Y(k)-Y(j)=\lambda \sum_{s \in T_{k}} Y(s),
$$

where $T_{k}$ is the component of $T-\{j\}$ that contains $k$. Since $T_{k}$ is a subgraph of $T_{j}$ containing at least one less vertex (that is, $j$ ) it follows that $Y(k)-Y(j)<$ $Y(j)-Y(i)$.

Now we are in a position to state the main result of this section.

TheOREM 3.5. Let $T$ be an unweighted tree, $L$ the Laplacian matrix of $T$. Let $Z$ be a 3-vector of $L$. Then either of the following occurs.

I. The cardinality of $\mathcal{C}(T, Z)$ is 1 . In this case $\mathcal{C}(T, Z)$ contains a vertex, say $k$, and the subgraph of $T$ induced by the set of vertices corresponding to the $0^{\prime}$ s in $Z$ is connected. The entries of $Z$ either increase and are concave down, decrease and are concave up or are identically zero along any path in $T$ which starts at $k$.

II. The cardinality of $\mathcal{C}(T, Z)$ is 2 . Then one of the following cases occur.

a. $\mathcal{C}(T, Z)=\{u, v\}$

Let $P$ be the path joining $u$ and $v$. Along any path in $T$ which starts at $u$ or $v$ and does not pass through any other vertices of $P$, the entries of $Z$ either increase and are concave down, decrease and are concave up or are identically zero. The entries of $Z$ along the path $P$ satisfy one of the following three descriptions.

1. Entries are identically zero. Along any path in $T$ which starts at a vertex on $P$ (not $u$ or $v$ ) and does not pass through any other vertices of $P$, the entries of $Z$ are 
identically zero.

2. Entries are positive (except for $u$ and $v$ ) and unimodal. Along any path in $T$ which starts at a vertex on $P$ (not $u$ or $v$ ) and does not pass through any other vertices of $P$, the entries of $Z$ increase and are concave down.

3. Entries are negative (except for $u$ and $v$ ) and entries of $-Z$ along the path $P$ are unimodal. Along any path in $T$ which starts at a vertex on $P$ (not $u$ or $v$ ) and does not pass through any other vertices of $P$, the entries of $Z$ decrease and are concave up.

b. $\mathcal{C}(T, Z)=\{u,[v, w]\}$.

Without loss of generality, assume that $v$ is the nearest vertex to $u$ and $Z(w)<0$. Let $P$ be the path joining $u$ and $v$. Along any path in $T$ which starts at $u$ and does not pass through any other vertices of $P$, the entries of $Z$ either increase and are concave down, decrease and are concave up or are identically zero. Along any path in $T$ which starts at $w$ and does not pass through any other vertices of $P$, the entries of $Z$ decrease and are concave up. The entries of $Z$ along the path $P$ are positive (except for $u$ ) and unimodal. Along any path in $T$ which starts at a vertex on $P$ (not $u$ ) and does not pass through any other vertices of $P$, the entries of $Z$ increase and are concave down. c. $\mathcal{C}(T, Z)=\{[x, u],[v, w]\}$.

Let the distance between $v$ and $u$ be less than the distance between $w$ and $x$ by 2 units. Let $Z(x)>0$. Thus it follows that ${ }^{1} Z(u)<0, Z(v)<0$ and $Z(w)>0$. Let $P$ be the path joining $u$ and $v$. Along any path in $T$ which starts at $x$ or $w$ and does not pass through any other vertices of $P$, the entries of $Z$ increase and are concave down. The entries of $Z$ along the path $P$ are negative and the entries of $-Z$ along the path $P$ are unimodal. Along any path in $T$ which starts at a vertex on $P$ and does not pass through any other vertices of $P$, the entries of $Z$ decrease and are concave up.

Proof. First note that since $Z$ is a 3 -vector, the cardinality of $\mathcal{C}(T, Z)$ is $\leq 2$ (by Corollary 3.2).

Proof of $I$. Let the cardinality of $\mathcal{C}(T, Z)$ be 1 . Suppose that $Z$ has no zero entry. Then by Proposition 3.1, we get that the number of eigenvalues of $L$ which are less than ${ }^{3} \lambda$ is same as the number of unordered pairs $(i, k)$ such that $L(i, k) Z(i) Z(k)>0$. But there are exactly two eigenvalues of $L$ less than ${ }^{3} \lambda$. Thus $\mathcal{C}(T, Z)$ must contain two edges, and this is a contradiction to the hypothesis. So $Z$ has a zero entry and so $T$ has a characteristic vertex $k$, (say). Since the cardinality of the characteristic set is $1, T-\{k\}$ is disconnected with at least two nonzero components and each of the components is either positive or negative or zero. Thus the subgraph of $T$ induced by the zero vertices is connected. The rest of the proof of this item follows by Lemma 3.4 .

Proof of II. We only prove item (a). The proof of other items are similar. Note that any nonzero component of $T-\{u\}$ is either positive or negative or contains a characteristic element and the only possible characteristic element is $v$. Thus each of the components, except for the one which contains $v$, of $T-\{u\}$ is either positive or negative or zero. Also note that the component which contains $v$ contains $P-\{u\}$ as

\footnotetext{
${ }^{1}$ It cannot be the case that $Z(x)>0, Z(u)<0, Z(v)>0$ and $Z(w)<0$, for then we must have another characteristic element lying on the path joining $u$ and $v$.
} 
a subgraph. Thus by Lemma 3.4, it follows that along any path in $T$ which starts at $u$ and does not pass through any other vertices of $P$, the entries of $Z$ either increase and are concave down, decrease and are concave up or are identically zero. The same is true for paths starting at $v$ and not passing through any other vertices of $P$.

If $u$ and $v$ are adjacent then we have nothing more to prove. So let $P=$ $\left[u, u_{1}, \cdots, u_{r}, v\right]$. Observe that each of the nonzero components of $T-\{u, v\}$ has to be either positive or negative; otherwise, it will lead to the presence of another characteristic element. Suppose that $H$ is the component of $T-\{u, v\}$ which contains the vertex $u_{1}$. If $H$ is a zero component we have nothing to prove. So, let $H$ be positive. If $P^{\prime}$ is any path starting at $u_{i}, i \in\{1,2, \cdots, r\}$ and not passing through any other vertices of $P$, then by Lemma 3.4, entries of $Z$ increase and are concave down along $P^{\prime}$.

It remains to show that along the path $P$ the entries of $Z$ are unimodal. Towards this, note that if $Z\left(u_{i}\right)<Z\left(u_{i-1}\right)$ then the eigencondition at the vertex $u_{i}$ implies that there must be a vertex $x$ adjacent to $u_{i}$ such that $Z(x)<Z\left(u_{i}\right)$. As $Z\left(u_{i}\right)<Z\left(u_{i-1}\right)$ and along any path starting at $u_{i}$ and not passing through any other vertices of $P$ the entries of $Z$ increase, we get that $x$ is $u_{i+1}$. In the same way one can conclude that $Z\left(u_{i+2}\right)<Z\left(u_{i+1}\right)$ and so on. Thus the proof of item (a) of (II) is complete. $\square$

Below we give some examples to show the occurrence of each of the cases described in the above theorem.

EXAMPLE 3.6. The graph in Fig. 3.2 is an unweighted tree. The third smallest eigenvalue of the Laplacian matrix is 0.1981 (rounded to four decimal places) and has multiplicity 3 . The eigenvector $Y_{1}$ corresponds to the case I, eigenvector $Y_{2}$ corresponds to the case II-(a)-(1) and eigenvector $Y_{3}$ corresponds to the case II-(a)-(2). The occurrence of II-(a)-(3) can be seen by considering $-Y_{2}$. The occurrence of case (II)-(b) is shown in the Example 3.7. One can notice the occurrence of case (II)-(c) in an unweighted path of 20 vertices. The entries of each of the vectors below are rounded to four decimal places.

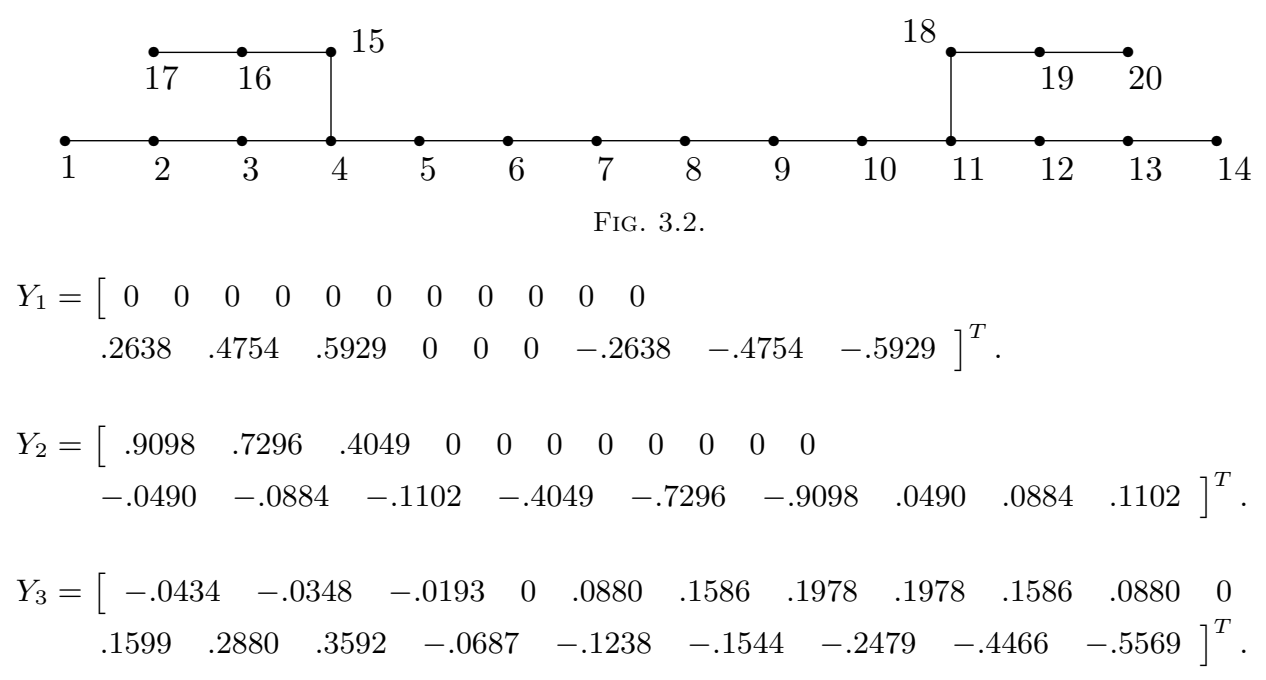


EXAMPLE 3.7. In this example we will show the occurrence of the case (II)(b) described in Theorem 3.5. Consider the weighted path on four vertices whose Laplacian matrix is given by $L$. The vector $Y$ corresponding to the third smallest eigenvalue of $L$ is given below.

$$
L=\left[\begin{array}{rrrr}
1 & -1 & 0 & 0 \\
-1 & 2 & -1 & 0 \\
0 & -1 & 1+\frac{3+\sqrt{5}}{2} & -\frac{3+\sqrt{5}}{2} \\
0 & 0 & -\frac{3+\sqrt{5}}{2} & \frac{3+\sqrt{5}}{2}
\end{array}\right] ; Y=\left[\begin{array}{r}
1 \\
-\frac{1+\sqrt{5}}{2} \\
0 \\
\frac{1+\sqrt{5}}{3+\sqrt{5}}
\end{array}\right]
$$

The third smallest eigenvalue is $\frac{3+\sqrt{5}}{2}$.

4. Multiplicity of ${ }^{3} \lambda$. Suppose $Y$ is a 3 -vector of a tree $T$. It is known (see, e.g., $[5$, corollary $(2,5)]$ ) that when each entry of a 3 -vector is different from zero, the eigenvalue ${ }^{3} \lambda$ is simple.

Thus we assume the existence of a 3 -vector $Y$ with at least one characteristic vertex.

Lemma 4.1. Suppose $Y$ is a 3-vector for a tree $T$ and that $u$ is a vertex in $\mathcal{C}(G, Y)$. Then there exists exactly one component $T_{0}$ of $T-\{u\}$ for which $\tau\left(L_{T_{0}}\right)<{ }^{3} \lambda$. There is at least one component $T_{1}$ of $T-\{u\}$ for which $\tau\left(L_{T_{1}}\right)={ }^{3} \lambda$. For the rest of the components $T_{2}, \cdots, \tau\left(L_{T_{i}}\right) \geq{ }^{3} \lambda$.

Further, if ${ }^{3} \lambda$ is not an eigenvalue of $L_{T_{0}}$ then there exists another component $T_{2}$ such that $\tau\left(L_{T_{2}}\right)={ }^{3} \lambda$.

Proof. Since $u$ is a characteristic vertex there are at least two nonzero components $C_{1}, C_{2}$ of $T-\{u\}$. Since $|\mathcal{C}(G, Y)| \leq 2$, it follows that at least one of $C_{1}$ or $C_{2}$ has to be negative or positive. Say, $C_{1}$ is positive. From the eigenvalue eigenvector relation and the fact that $Y(u)=0$, it follows now that $\tau\left(L_{C_{1}}\right)={ }^{3} \lambda$.

Let $T_{i}$ be the components of $T-\{u\}$ arranged in the order such that $\tau\left(L_{T_{i}}\right) \leq$ $\tau\left(L_{T_{i+1}}\right) ; i=0,1,2, \cdots$. If $\tau\left(L_{T_{i}}\right) \geq \alpha$ for each $i$ then by the Cauchy interlacing theorem we have that the algebraic connectivity of $T$ is at least $\alpha$. By hypothesis ${ }^{3} \lambda$ is strictly larger than the algebraic connectivity. Thus $\tau\left(L_{T_{0}}\right)<{ }^{3} \lambda$. Further if $\tau\left(L_{T_{0}}\right) \leq \tau\left(L_{T_{1}}\right)<{ }^{3} \lambda$, then consider the components $T_{0}, T_{1}, C_{1}$ (in the first paragraph we have proved the existence of a component $C_{1}$ such that $\left.\tau\left(L_{C_{1}}\right)={ }^{3} \lambda\right)$ and apply Lemma 2.6 to arrive at a contradiction. Thus $\tau\left(L_{T_{1}}\right)={ }^{3} \lambda$.

Note that ${ }^{3} \lambda$ is an eigenvalue of $L_{C_{2}}$ (under discussion in the first paragraph) with the corresponding eigenvector $Y\left(C_{2}\right)$. If ${ }^{3} \lambda$ is not an eigenvalue of $L_{T_{0}}$ then the component $C_{2} \neq T_{0}$. Since $T_{0}$ is the only component with $\tau\left(L_{T_{0}}\right)<{ }^{3} \lambda$, we have $\tau\left(L_{C_{2}}\right)={ }^{3} \lambda$. प

THEOREM 4.2. Suppose $Y$ is a 3 -vector for a tree $T$ and a vertex $u \in \mathcal{C}(G, Y)$. Let $T_{0}, T_{1}, \cdots$ be the components of $T-\{u\}$ such that $\tau\left(L_{T_{0}}\right)<{ }^{3} \lambda, \tau\left(L_{T_{1}}\right)=\tau\left(L_{T_{2}}\right)=$ $\cdots=\tau\left(L_{T_{k}}\right)={ }^{3} \lambda$ and $\tau\left(L_{T_{i}}\right)>{ }^{3} \lambda, \forall i>k$.

Then mult ${ }_{3 \lambda}(L)=k-1+$ mult $_{3_{\lambda}}\left(L_{T_{0}}\right)$.

Proof of Case 1. If ${ }^{3} \lambda$ is not an eigenvalue of $L_{T_{0}}$ then we know by Lemma 4.1 $k \geq 2$. Further, for any 3 -vector $X$ of $L, X\left(T_{0}\right)=0$. (By Lemma $2.3 X(u)=0$. If $X\left(T_{0}\right) \neq 0$, then it would follow that ${ }^{3} \lambda$ is an eigenvalue of $L_{T_{0}}$.) 
For $i=1, \cdots, k-1$, let $Z_{i, k}$ be the vector such that $Z_{i, k}\left(T_{i}\right)$ is the positive eigenvector of $L_{T_{i}},-Z_{i, k}\left(T_{k}\right)$ is the positive eigenvector of $L_{T_{k}} ; Z_{i, k}$ is zero elsewhere and the sum of entries of $Z_{i, k}$ is zero.

It is now easy to see that the 3 -vector $X$ is really a vector in the span of the linearly independent set of 3 -vectors $\left\{Z_{i, k}: i=1, \cdots, k-1\right\}$. Thus we get mult $_{3_{\lambda}}(L)=$ $k-1+$ mult $_{3 \lambda}\left(L_{T_{0}}\right)$.

Proof of Case 2. Suppose ${ }^{3} \lambda$ is an eigenvalue of $L_{T_{0}}$ and $W_{1}, \cdots, W_{r}$ are the independent eigenvectors of $L_{T_{0}}$ corresponding to ${ }^{3} \lambda$. For $j=1, \cdots, r$ define

$$
Z^{j, k}=\left\{\begin{array}{cl}
W_{j} & \text { on } T_{0} \\
\text { the positive eigenvector of } L_{T_{k}} & \text { on } T_{k} \\
0 & \text { elsewhere }
\end{array}\right.
$$

normalized so that the sum of entries is zero. Then it is routine to see that the set of vectors $\left\{Z^{j, k}, Z_{i, k}: j=1, \cdots, r ; i=1, \cdots, k-1\right\}$ is a linearly independent set of 3 -vectors for $L$ and any 3 -vector $X$ is really a linear combination of these vectors. Thus mult $_{{ }_{3}}(L)=k-1+$ mult $_{3_{\lambda}}\left(L_{T_{0}}\right)$.

Acknowledgement. The author thanks the referee for some valuable suggestions.

\section{REFERENCES}

[1] R. B. Bapat and S. Pati. Algebraic connectivity and the characteristic set of a graph. Linear and Multilinear Algebra, 45:247-273, 1998.

[2] S. Fallat and S. Kirkland. Extremizing algebraic connectivity subject to graph theoretic constraints. Electronic Journal of Linear Algebra, 3:48-74, 1998.

[3] S. Fallat, S. Kirkland, and S. Pati. Minimizing algebraic connectivity over connected graphs with fixed girth. Discrete Mathematics, to appear.

[4] M. Fiedler. Algebraic connectivity of graphs. Czechoslovak Mathematical Journal, 23:298-305, 1973.

[5] M. Fiedler. Eigenvectors of acyclic matrices. Czechoslovak Mathematical Journal, 25:607-618, 1975.

[6] M. Fiedler. A property of eigenvectors of nonnegative symmetric matrices and its application to graph theory. Czechoslovak Mathematical Journal, 25:619-633, 1975.

[7] M. Fiedler. Laplacian of graphs and algebraic connectivity. Combinatorics and graph theory, Banach Centre Publications, Warsaw, 25:57-70, 1989.

[8] R. Grone and R. Merris. Algebraic connectivity of trees. Czechoslovak Mathematical Journal, 37:660-670, 1987.

[9] R. Grone, R. Merris, and V. S. Sunder. The Laplacian spectrum of a graph. SIAM Journal on Matrix Analysis and Applications, 11:218-238, 1990.

[10] S. Kirkland and S. Fallat. Perron components and algebraic connectivity for weighted graphs. Linear and Multilinear Algebra, 44:131-148, 1998.

[11] S. Kirkland, M. Neumann, and B. L. Shader. Characteristic vertices of weighted trees via Perron values. Linear and Multilinear Algebra, 40:311-325 1996.

[12] S. Kirkland, M. Neumann, and B. L. Shader. Distances in weighted trees and group inverses of Laplacian matrices. SIAM Journal on Matrix Analysis and Applications, 18:827-841, 1997.

[13] R. Merris. Characteristic vertices of trees. Linear and Multilinear Algebra, 22:115-131, 1987.

[14] R. Merris. Laplacian matrices of graphs: a survey. Linear Algebra and its Applications, 197:143$176,1994$.

[15] R. Merris. A survey of graph Laplacians. Linear and Multilinear Algebra, 39:19-31, 1995. 


\section{ELA}

[16] B. Mohar. Laplace eigenvalues of graphs-a survey. Discrete Mathematics, 109:171-183, 1992.

[17] Y. L. Pan, J. S. Li, and Y. P. Hou. Lower bounds on the smallest Laplacian eigenvalue of a graph. Linear and Multilinear Algebra, to appear. 